\title{
Clinical characteristics of patients with benign nonlesional temporal lobe epilepsy
}

\author{
This article was published in the following Dove Press journal: \\ Neuropsychiatric Disease and Treatment \\ 28 July 2016 \\ Number of times this article has been viewed
}

\author{
Jiyeon Kim' \\ Seong Hoon Kim² \\ Sung Chul Lim² \\ Woojun $\mathrm{Kim}^{2}$ \\ Young-Min Shon ${ }^{3}$
}

'Department of Neurology, Korea University Ansan Hospital, College of Medicine, Korea University, Ansan, ${ }^{2}$ Department of Neurology, Catholic Neuroscience Institute, College of Medicine, The Catholic University of Korea, Seocho-gu, ${ }^{3}$ Department of Neurology, Samsung Medical Center, Sungkyunkwan University School of Medicine, Seoul, Korea
Correspondence: Young-Min Shon Department of Neurology, Samsung Medical Center, Sungkyunkwan University School of Medicine, 8I Irwonro, Gangnam-gu, Seoul |35-7|0, Korea

Tel +82234102701

Fax +82234100052

Email sonogung@gmail.com
Purpose: To evaluate the evolution of nonlesional temporal lobe epilepsy (TLE-NL) in patients treated exclusively with antiepileptic drugs and to elucidate clinical phenotypes related to the prognosis of these patients.

Methods: Clinical, radiological, and electroencephalographic (EEG) findings in 84 patients with TLE-NL were reviewed. A good response group (GRG) and a poor response group (PRG) were defined if the duration of their seizure-free period was $>1$ year, or $<1$ year, respectively.

Results: There were 46 (54.8\%) patients in the GRG and 38 (45.2\%) patients in the PRG. The number of antiepileptic drugs administered was significantly lower in the GRG than that in the PRG $(1.3 \pm 0.8$ vs $2.8 \pm 1.0$, respectively; $P<0.05)$. The GRG had a significantly older age of onset than the PRG and a lower occurrence of initial precipitating events, such as febrile seizures, central nervous system infection, and head trauma $(P<0.05)$. The prevalence of EEG abnormality, presence of aura, generalized seizures, and automatism was less frequently observed in the GRG $(P<0.05)$. Multivariate analysis showed that the presence of automatism and initial precipitating events were significantly associated with a poor prognosis $(P<0.05)$.

Conclusion: In contrast to the commonly assumed intractability of TLE, we found that more than $54 \%$ of patients with TLE-NL achieved a long seizure-free period. Older age at onset of TLE-NL was associated with a better prognosis. However, the presence of automatism and initial precipitating events were related to a poor prognosis. Future prospective studies with a much larger population are warranted.

Keywords: epilepsy, benign temporal lobe epilepsy, nonlesional temporal lobe epilepsy

\section{Introduction}

Epilepsy is a common and disabling condition. Medical treatment fails to control seizures in approximately $20 \%-30 \%$ of patients. ${ }^{1}$ Temporal lobe epilepsy (TLE) is the most frequent medically refractory type of epilepsy syndrome seen in epilepsy clinics. According to the classification of the International League against Epilepsy, ${ }^{2}$ the diagnosis of TLE encompasses patients with similar seizure semiology and electroencephalographic (EEG) characteristics that point to an ictal onset zone in the temporal lobe. TLE accounts for $\sim 40 \%$ of all patients with partial seizures or $15 \%-20 \%$ of all patients with epilepsy. 3,4

TLE has a variety of different etiologies. The most common etiology is hippocampal sclerosis (HS), which is responsible for $60 \%-70 \%$ of all TLE cases. Individuals with TLE-HS have a typical clinical presentation, frequent occurrences of pharmaco-resistance, and good surgical outcomes. ${ }^{5,6}$ Different structural etiologies, such as tumors, focal cortical dysplasias, and vascular or ischemic lesions, account for $10 \%-15 \%$ of all TLE cases. There are no detectable structural lesions, even using 
modern magnetic resonance imaging (MRI) protocols, in $15 \%-20 \%$ of individuals with TLE. ${ }^{7}$

Nonlesional TLE (TLE-NL) is a heterogeneous disorder in that some patients have a severe refractory form, whereas many others have a mild epileptic disorder and enter remission with or without antiepileptic drug (AED) administration. ${ }^{8}$ Indeed, the ability to predict the clinical course of this type of epilepsy would help both physicians and patients. Clinical aspects of TLE-HS have been studied to a great extent, but analyses of AED response and prognosis of TLE-NL patients are lacking. Therefore, it is not clear why the effect of AEDs within this group is so variable, ranging from a complete seizure-free state under a minimal dose of AED monotherapy to a refractory condition that leads to the need for surgical resection of the temporal lobe.

In this study, we examined how clinical and electrophysiological phenotypes relate to the prognosis of patients with TLE-NL who were treated exclusively with AEDs.

\section{Methods}

\section{Patients}

Patients with TLE-NL who were investigated from March 2006 to April 2014 at either Seoul St Mary’s Hospital or Yeouido St Mary's Hospital, located in Seoul, Korea, were included in the study. The diagnosis of TLE was made on the basis of reliable indicators, including a range of clinical seizure semiology, EEG, and MRI criteria. ${ }^{2}$ Data collected at enrollment included patient demographics, family history of epilepsy, age at onset, presence of initial precipitating insults (eg, febrile seizures, central nervous system [CNS] infection, head trauma), seizure semiology and frequency, neurological and cognitive findings, history of AED usage, interictal EEGs, and imaging findings. All of the patients enrolled had a history of two or more unprovoked seizures, regardless of age of onset, and had been followed up for at least 1 year. The interictal EEG studies were always performed using video-EEG monitoring or routine sleep EEG, with $>30$-minute recordings. The epileptiform discharges (EDs) were exclusively involved in the temporal region as indicated by their occurrence over electrodes F7, F8, T3, and T4, and supplementary electrodes $\mathrm{T} 1$ and T2. The EDs were labeled as unilateral if confined to one side (left temporal: T1, F7, T3; right temporal: T2, F8, T4) at least $95 \%$ of the time. Any implication of seizure onset outside the mesial temporal structures by seizure semiology (eg, auditory, visual aura, or hemifacial sensory aura) or EEG features (eg, EDs on T5 or T6) was a criterion for exclusion. In addition, patients with incomplete clinical history, poor compliance, or psychogenic seizures and those who were lost to follow-up were excluded from the study. This study was approved by the local ethics committee, and all subjects provided written informed consent, in accordance with the guidelines of St Mary's Hospital in Seoul, Republic of Korea.

\section{Statistical analysis}

Patients were classified on the basis of univariate and multivariate analyses into the good response group (GRG) and the poor response group (PRG) if the duration of their seizure-free period was $>1$ year or $<1$ year, respectively. The Student's $t$-test for unpaired samples was used to assess differences between groups for continuous variables. Categorical variables were expressed as percentages, and differences among group distributions were assessed using the chi-square test. For multivariate analysis, tested covariates included age at onset of epilepsy, initial precipitating events, ictal manifestations (eg, presence of aura, automatism, and generalized seizure), and EEG abnormality. Variables that were associated with poor prognosis were identified using univariate analysis and a significance level of 5\%. These variables were then included in a multivariate logistic regression model. All calculations were done by SPSS version 18 software (SPSS Inc., Chicago, IL, USA).

\section{Results}

\section{Patient recruitment}

A total of 200 patients were assessed. Eighty-four patients (42\%) fulfilled our inclusion criteria, whereas 116 were excluded for one or more of the following reasons: lost to follow-up ( $\mathrm{n}=28)$, psychogenic or other nonepileptic seizures $(\mathrm{n}=5)$, poor compliance $(\mathrm{n}=21)$, and incomplete medical records $(n=62)$.

\section{Clinical and instrument findings}

The sample included 84 patients (41 women and 43 men; Table 1) with TLE-NL. The mean \pm standard deviation (SD) age at study entry was $42.1 \pm 15.1$ years, and age at onset of epilepsy was $25.1 \pm 16.7$ years. Four patients $(4.8 \%)$ had a family history of epilepsy and eight patients $(9.5 \%)$ had precipitating events. Fifty-nine patients $(70.2 \%)$ showed one or more of four types of aura (30 [35.7\%] had epigastric aura, 30 [35.7\%] had psychic aura, five [6.0\%] had special sensory symptoms [olfactory or gustatory aura], and 28 [33.3\%] had unclassified vague symptoms like dizziness, dullness, or a "woozy" feeling; Table 2). Complex partial seizures (loss of consciousness or unresponsiveness) were found in 65 patients $(78.3 \%)$ and were associated with 
Table I Demographic data of the present study

\begin{tabular}{|c|c|c|c|c|}
\hline Demographic data of patients & GRG ( $\mathbf{N}=46)$ & PRG (N=38) & Total $(\mathbf{N}=\mathbf{8 4})$ & $P$-value \\
\hline Age at enrollment (years), mean (SD) & $45.4(15.8)$ & $38.1(13.3)$ & $42.1(15.1)$ & $0.027(0.2)^{\ddagger}$ \\
\hline Age at epilepsy onset (years), mean (SD) & $28.6(18.7)$ & $20.9(12.8)$ & $25.1(16.7)$ & $0.027(0.1)^{\ddagger}$ \\
\hline Duration of epilepsy (years), mean (SD) & $16.8(10.0)$ & $18.2(14.0)$ & I7.4 (II.9) & NS \\
\hline Sex (male), n (\%) & $21(45.7)$ & $22(57.9)$ & $43(51.2)$ & \\
\hline Positive family history, n (\%) & $\mathrm{I}(2.2)$ & $3(7.9)$ & $4(4.8)$ & \\
\hline Initial precipitating insults,* $\mathrm{n}(\%)$ & $\mathrm{I}(2.2)$ & $7(18.4)$ & $8(9.5)$ & $0.012(0.031)^{\ddagger}$ \\
\hline Number of AED, mean (SD) & $\mathrm{I} .33(0.79)$ & $2.89(0.95)$ & $2.04(1.16)$ & $<0.001$ \\
\hline
\end{tabular}

Notes: *Initial precipitating insults: febrile seizures, CNS infection, and head trauma. ¥Multivarivate logistic regression.

Abbreviations: AED, antiepileptic drug; CNS, central nervous system; GRG, good response group; NS, nonsignificant; PRG, poor response group; SD, standard deviation.

oroalimentary or hand automatisms in 38 patients $(45.2 \%)$, versive seizures in 18 patients $(21.4 \%)$, and secondary generalization (major motor seizures) in 60 patients $(71.4 \%)$. A normal interictal EEG was found in three patients (3.6\%). Epileptiform abnormalities (spike or sharp wave) were found in 75 patients $(89.3 \%)$, and focal slow activities were observed in only six patients $(7.1 \%)$.

\section{Prognostic predictors}

As shown in Table 1, there were $46(54.8 \%)$ patients in the GRG and 38 (45.2\%) patients in the PRG. Patients in the GRG had seizure-free periods with a mean duration of 52 months (4.3 years). The average number of AEDs was significantly lower in the GRG (1.3 \pm 0.8$)$ than in the PRG $(2.8 \pm 1.0$; $P<0.05)$. Compared with patients in the PRG, patients in the GRG had a significantly older age at onset of epilepsy (28.6 \pm 18.6 vs $20.8 \pm 12.7$ years; $P<0.05$ ), and a lower occurrence of initial precipitating events like febrile seizures, CNS infection, and head trauma $(2.2 \%$ vs $18.4 \% ; P<0.05)$. Among clinical and EEG variables, automatism was less frequently observed in the GRG than in the PRG (30.4\% vs $63.2 \%$, respectively; $P<0.01$; Table 2 ). Multivariate analysis showed that the presence of automatism and initial precipitating events negatively affected the prognosis. Both variables were significantly associated with a poor prognosis $(P<0.05)$. There were no additional meaningful differences in other variables between the two groups of patients with TLE-NL.

\section{Discussion}

The most common focal epilepsy is TLE and the most frequent underlying pathology is HS. The combination of typical seizure semiology, radiological findings, interictal and ictal EEG findings, and the neuropsychological profiles of patients with TLE-HS usually provides sufficient evidence to support an anterior temporal lobectomy, which in turn leads to excellent postoperative seizure outcomes. ${ }^{9}$ However, TLE-NL is a disease entity that is not yet as thoroughly studied as TLE-HS.

Table 2 Clinical characteristics of the patients in the present study

\begin{tabular}{|c|c|c|c|c|}
\hline Variable & GRG $(\mathbf{N}=46)$ & PRG $(\mathbf{N}=38)$ & Total $(\mathbf{N}=\mathbf{8 4})$ & $P$-value \\
\hline Laterality & & & & NS \\
\hline $\mathrm{R}$ & $12(26.1)$ & $9(23.7)$ & $21(25.0)$ & \\
\hline L & $25(54.3)$ & $23(60.5)$ & $48(57.1)$ & \\
\hline B & $9(19.6)$ & $6(15.8)$ & $15(17.9)$ & \\
\hline Any type of aura & $30(65.2)$ & $29(76.3)$ & $59(70.2)$ & NS \\
\hline Epigastric aura & $13(28.3)$ & $17(44.7)$ & $30(35.7)$ & \\
\hline Psychic aura & $14(30.4)$ & $16(42.1)$ & $30(35.7)$ & \\
\hline Special sensory symptoms (olfactory, gustatory aura) & $3(6.5)$ & $2(5.3)$ & $5(6.0)$ & \\
\hline Unclassified & $12(26.1)$ & $16(42.1)$ & $28(33.3)$ & \\
\hline Automatism & $14(30.4)$ & $24(63.2)$ & $38(45.2)$ & $0.003(0.004)^{\ddagger}$ \\
\hline Versive seizure & $8(17.4)$ & $10(26.3)$ & $18(21.4)$ & NS \\
\hline Complex partial seizure & $34(75.6)$ & $31(81.6)$ & $65(78.3)$ & \\
\hline Secondarily generalized & $30(65.2)$ & 30 (78.9) & $60(71.4)$ & \\
\hline \multicolumn{5}{|l|}{ Interictal EEG } \\
\hline Normal & $2(4.3)$ & I (2.6) & $3(3.6)$ & \\
\hline Abnormal, spikes, or sharp waves & $40(86.9)$ & $33(86.8)$ & $75(89.3)$ & \\
\hline Abnormal, focal slow & $4(8.7)$ & $2(5.3)$ & $6(7.1)$ & \\
\hline
\end{tabular}

Notes: Values are expressed as n (\%). ${ }^{*}$ Multivarivate logistic regression.

Abbreviations: B, bilateral; EEG, electroencephalogram; GRG, good response group; L, left; NS, nonsignificant; PRG, poor response group; R, right. 
Epilepsies of unknown origin are known to be controlled more easily with medications than epilepsies with structural lesions, ${ }^{10}$ so it is reasonable to assume that a significantly larger proportion of TLE-NL than TLE-HS patients would experience more adequate seizure control with AEDs than the TLE-HS patients. Studies on the prognosis of TLE-NL are still limited, so this assumption has not been confirmed to date.

In a previous cross-sectional study of 73 nonsurgical adult patients with TLE with or without HS, approximately two-thirds were found to have a good prognosis after a follow-up ranging from 36 to 96 months. ${ }^{8}$ More recently, studies by Labate et al ${ }^{11,12}$ provide evidence about the existence of a common, often unrecognized clinical entity called benign TLE. Benign TLE is characterized by seizure onset in adulthood, rare simple partial seizures, and radiological evidence of HS in almost $40 \%$ of the patients studied. However, as previously mentioned, these studies included patients with TLE-HS, and it is well known that TLE-HS shows severe and widespread structural abnormalities. Hence, studies about the MRI-negative TLE are rare and needed.

We found that more than $54 \%$ of patients with TLE-NL can achieve a long seizure-free period with AEDs and that this pattern was significantly more prevalent in patients with older age at seizure onset. Previous studies have identified the association of early age of epilepsy onset with poor seizure outcomes. ${ }^{13,14}$ On the other hand, the present data showed a direct association of either the presence of automatism during seizures or initial precipitating events with a poor prognosis. The incidence of automatisms in our patient group is similar to that of other studies, ${ }^{15,16}$ regardless of differences in study populations and methods. Previous studies showed an increased incidence of automatisms in longer seizures. ${ }^{17}$ In addition, oral and hand automatisms have been reported to be provoked by stimulating the anterior cingulate gyrus and mesial frontal structures, ${ }^{18}$ indicating that automatisms may be generated by spreading ictal activity from extratemporal areas. Therefore, it can be assumed that the poor prognosis in patients with automatism may have more widespread and longer ictal-interictal EDs, which inevitably induce irreversible brain damage to the patient.

Another study suggested that a positive family history is clinically significant and associated with a favorable outcome in mild TLE-NL. ${ }^{8}$ In addition, a genetic predisposition for benign TLE has been suggested on the basis of similar electroclinical and neuroimaging features in many familial cases. ${ }^{11}$ In contrast, the incidence of family history in our study is lower than the aforementioned studies, and there was no meaningful difference between the two groups with regard to patient family history. These differences may be because of insufficient information about family history or the variations in the study populations, especially the presence of TLE-HS patients in the previous studies.

In the data of our patients, laterality of ictal onset was usually unilateral (more than $80 \%$ ) and more frequently located over left temporal region. Like Aguglia et al, ${ }^{13}$ we did not find any significant differences between our GRG and PRG patients in terms of the laterality.

Our study has some limitations. First, the diagnosis of TLE-NL was based predominantly on seizure descriptions, EEGs, and routine MRIs (quantitative analysis of hippocampal volume, like MRI volumetry, could not be obtained for our patients). Also, the patients in our study were recruited from two different epilepsy centers, leaving the possibility of nonhomogeneous patient inclusion criteria. However, the MRI was accomplished with 1.5 or $3 \mathrm{~T}$ and the same protocol in all cases, and the images were all reviewed scrutinized by the same experienced neurologist and radiologist (JH Jang and $\mathrm{W}$ Kim).

Second, we could not obtain video-EEG monitoring results from all of our patients, thus preventing the detection of exact seizure semiology, including automatisms and ictal EEG patterns. It must be emphasized, however, that the diagnosis of TLE in all of our patients was made on the basis of a constellation of clinical, MRI, and EEG criteria that are considered to be reliable interictal indicators of TLE. ${ }^{19}$

Lastly, because our study was retrospective, we investigated a highly selective patient group of TLE patients who had been recruited and evaluated at only two tertiary epilepsy centers. This bias should be eliminated in the future by executing prospective, well-controlled studies with a much larger population.

\section{Conclusion}

The present study provides evidence for a new perspective on the care of patients with TLE-NL. Our results suggest that an older age at onset of TLE-NL predicts good seizure control with AED treatment. In addition, it may be hard to achieve remission in TLE-NL patients with automatism or with initial precipitating insult, such as febrile seizure.

\section{Disclosure}

The authors report no conflicts of interest in this work. 


\section{References}

1. Cockerell OC, Johnson AL, Sander JW, Hart YM, Shorvon SD Remission of epilepsy: results from the National General Practice Study of Epilepsy. Lancet. 1995;346(8968):140-144.

2. [No authors listed]. Proposal for revised classification of epilepsies and epileptic syndromes. Commission on Classification and Terminology of the International League Against Epilepsy. Epilepsia. 1989;30(4): 389-399.

3. Benbadis SR. Is the underlying cause of epilepsy a major prognostic factor for recurrence? Neurology. 1999;53(2):440.

4. Löscher W. Current status and future directions in the pharmacotherapy of epilepsy. Trends Pharmacol Sci. 2002;23(3):113-118.

5. Wieser HG; ILAE Commission on Neurosurgery of Epilepsy. ILAE Commission Report. Mesial temporal lobe epilepsy with hippocampal sclerosis. Epilepsia. 2004;45(6):695-714.

6. Wieshmann UC, Larkin D, Varma T, Eldridge P. Predictors of outcome after temporal lobectomy for refractory temporal lobe epilepsy. Acta Neurol Scand. 2008;118(5):306-312.

7. Shukla G, Prasad AN. Natural history of temporal lobe epilepsy: antecedents and progression. Epilepsy Res Treat. 2012;2012:195073.

8. Aguglia U, Gambardella A, Le Piane E, et al. Mild non-lesional temporal lobe epilepsy. A common, unrecognized disorder with onset in adulthood. Can J Neurol Sci. 1998;25(4):282-286.

9. Elsharkawy AE, Alabbasi AH, Pannek H, et al. Long-term outcome after temporal lobe epilepsy surgery in 434 consecutive adult patients. $J$ Neurosurg. 2009;110(6):1135-1146.

10. Semah F, Picot MC, Adam C, et al. Is the underlying cause of epilepsy a major prognostic factor for recurrence? Neurology. 1998;51(5): $1256-1262$.
11. Labate A, Gambardella A, Andermann E, et al. Benign mesial temporal lobe epilepsy. Nat Rev Neurol. 2011;7(4):237-240.

12. Labate A, Ventura P, Gambardella A, et al. MRI evidence of mesial temporal sclerosis in sporadic "benign" temporal lobe epilepsy. Neurology. 2006;66(4):562-565.

13. Aguglia U, Beghi E, Labate A, et al. Age at onset predicts good seizure outcome in sporadic non-lesional and mesial temporal sclerosis based temporal lobe epilepsy. J Neurol Neurosurg Psychiatry. 2011;82(5): 555-559.

14. Beghi E, Tognoni G. Prognosis of epilepsy in newly referred patients: a multicenter prospective study. Collaborative Group for the Study of Epilepsy. Epilepsia. 1988;29(3):236-243.

15. Manford M, Fish DR, Shorvon SD. An analysis of clinical seizure patterns and their localizing value in frontal and temporal lobe epilepsies. Brain. 1996;119(Pt 1):17-40.

16. Janszky J, Fogarasi A, Magalova V, et al. Unilateral hand automatisms in temporal lobe epilepsy. Seizure. 2006;15(6):393-396.

17. Sadleir LG, Scheffer IE, Smith S, Connolly MB, Farrell K. Automatisms in absence seizures in children with idiopathic generalized epilepsy. Arch Neurol. 2009;66(6):729-734.

18. Talairach J, Bancaud J, Geier S, et al. The cingulate gyrus and human behaviour. Electroencephalogr Clin Neurophysiol. 1973;34(1):45-52.

19. Proposal for revised clinical and electroencephalographic classification of epileptic seizures. From the Commission on Classification and Terminology of the International League Against Epilepsy. Epilepsia. 1981;22(4):489-501.
Neuropsychiatric Disease and Treatment

\section{Publish your work in this journal}

Neuropsychiatric Disease and Treatment is an international, peerreviewed journal of clinical therapeutics and pharmacology focusing on concise rapid reporting of clinical or pre-clinical studies on a range of neuropsychiatric and neurological disorders. This journal is indexed on PubMed Central, the 'PsycINFO' database and CAS,

\section{Dovepress}

and is the official journal of The International Neuropsychiatric Association (INA). The manuscript management system is completely online and includes a very quick and fair peer-review system, which is all easy to use. Visit http://www.dovepress.com/testimonials.php to read real quotes from published authors. 